\title{
Laser na implantodontia: revisão integrativa
}

\author{
Laser in implantology: integrative review \\ Láser en implantología: revisión integrativa
}

Recebido: 25/02/2021 | Revisado: 04/03/2021 | Aceito: 09/03/2021 | Publicado: 17/03/2021

\author{
Ana Lethícia Leão Santos \\ ORCID: https://orcid.org/0000-0002-8888-3711 \\ Faculdade de Odontologia de Pernambuco, Brasil \\ E-mail: lethicia.leao@upe.br \\ Raquel Cavalcanti Carvalho Novaes \\ ORCID: https://orcid.org/0000-0003-1012-4480 \\ Faculdade de Odontologia de Pernambuco, Brasil \\ E-mail: raquel.carvalho@upe.br \\ Esdras Gabriel Alves-Silva \\ ORCID: https://orcid.org/0000-0002-2309-1115 \\ Universidade Estadual de Campinas, Brasil \\ E-mail: esdras0702@yahoo.com.br \\ Eloiza Leonardo de Melo \\ ORCID: https://orcid.org/0000-0001-5845-1478 \\ Faculdade de Odontologia de Pernambuco, Brasil \\ E-mail: eloiza.leonardo@upe.br \\ Marleny Elizabeth Márquez de Martínez Gerbi \\ ORCID: https://orcid.org/0000-0001-9174-2541 \\ Faculdade de Odontologia de Pernambuco, Brasil \\ E-mail: marleny.gerbi@upe.br \\ Mávio Eduardo Azevedo Bispo \\ ORCID: https://orcid.org/0000-0002-3781-0272 \\ Universidade de Pernambuco, Brasil \\ E-mail: meb@ecomp.upe.br \\ Renata Araújo Gomes de Sá \\ ORCID: https://orcid.org/0000-0001-6550-8225 \\ Faculdade de Odontologia de Pernambuco, Brasil \\ E-mail: renata.agsa@gmail.com \\ Maria Regina Almeida de Menezes \\ ORCID: https://orcid.org/0000-0003-3012-3979 \\ Faculdade de Odontologia de Pernambuco, Brasil \\ E-mail: regina.menezes@upe.br
}

\begin{abstract}
Resumo
No pós-operatório de um implante dental, o paciente pode experimentar vários sintomas de inflamação, tais como: inchaço, dor ao mastigar, sangramento e a formação de pus na área do implante. Nesse contexto, o uso do laser na implantodontia é um processo que busca melhoria na técnica de implante dentário, promovendo uma osseointegração mais efetiva do implante devido ao seu poder bioestimulante, acelerando, assim, a cicatrização. O objetivo deste trabalho foi realizar uma revisão integrativa da literatura atual sobre o uso de laser na implantodontia, destacando benefícios, limitações, desafios, relevâncias e impactos para saúde bucal e para sociedade. Como o uso do laser auxilia o processo de osseointegração na implantodontia? A revisão integrativa da literatura foi realizada por meio de uma busca de artigos científicos nas bases de dados eletrônica PubMed, Lilacs e Scopus utilizando-se dos descritores: “dental implant", "low level laser therapy" e "osseointegration", indexados no período de 2016 a 2020 que tratavam de clinical trial, review, systematic review, meta-analysis e randomized controlled e foram excluídos artigos de books and documents, confere paper, book chapter e editoriais. Após os critérios de elegibilidade foram analisados 15 (quinze) artigos integralmente publicados em Língua Inglesa. Os estudos mostraram que o uso do laser de baixa potência auxilia na implantodontia no que se refere a osseointegração do implante, diminuindo o período de pós-operatório, dor e risco de inflamação devido ao seu poder bioestimulante, analgésico e anti-inflamatório. Essa eficácia deriva de um aumento na proliferação epitelial e de fibroblasto, de um estímulo da síntese de colágeno, da atividade fagocítica e da indução de endorfina. Contudo, ainda não existem estudos de casos suficientes, principalmente em humanos, para que se comprove de fato que a utilização do laser auxilie no processo de integração osso-implante que é chamado de ósseointegração, entretanto, nota-se, que essa técnica se mostra promissora para a área da odontologia.
\end{abstract}

Palavras-chave: Implante dentário; Terapia a laser de baixo nível; Osseointegração. 


\begin{abstract}
In the postoperative period of a dental implant, the patient might experience several inflammation symptoms such as: swelling, pain when chewing, bleeding and secretion formation in the implanted area. In this context, the use of laser in implantology is a procedure that improves the technique of dental implant promoting an effectively implant osseointegration due to its biostimulant potential stimulating healing. The objective of this work was to carry out an integrative review of the current literature about the use of laser in implantology, highlighting the benefits, limitations, challenges, and its relevancy and impact on oral health and society. How the application of laser supports the process of osseointegration in implantology? An integrative review had been done through scientific articles in the databases PubMed, Lilacs e Scopus, using the descriptors "dental implant", "low level laser therapy" and "osseointegration", indexed from 2016 to 2020 which dealt with clinical trial, review, systematic review, meta-analysis and randomized controlled. There were excluded articles from books, documents, conference paper, book chapters and editorials. After the eligibility criteria there were analyzed 15 articles mainly published in English. The studies have shown that the application of a low level laser helps implant dentistry regarding the osseointegration of the implant, decreasing the postoperative period, pain and risk of infection due to its biostimulant potential, analgesic and anti-inflammatory. This effectiveness stems from an increase in epithelial and fibroblast proliferation, a stimulus of collagen synthesis, phagocytic activity and endorphin induction. Nevertheless, there are not yet enough case studies, especially in human beings to provoke, indeed the application of laser during the bone-implant integration process, which is called osseointegration. However, it is notable that this technique shows to be promissory in the dentistry industry.
\end{abstract}

Keywords: Dental implant; Low level laser therapy; Osseointegration.

\title{
Resumen
}

En el postoperatorio de un implante dental, el paciente puede sentir varios síntomas de inflamación, tales como: hinchazón, dolor al masticar, sangrado y la formación de pus en el área del implante. En este contexto, el uso del láser en la implantología dental es un proceso que busca la mejora en la técnica del implante dental, promoviendo una osteointegración más efectiva del implante debido a su poder bioestimulante, acelerando, de esta manera, la cicatrización. El objetivo de este trabajo fue realizar una revisión integral de la literatura actual sobre el uso del láser en el implante dental, destacando beneficios, limitaciones, desafíos, relevancias e impactos para la salud bucal y para la sociedad. ¿Cómo el uso del láser auxilia el proceso de osteointegración en la implantología dental? La revisión integral de la literatura fue realizada por medio de una búsqueda de artículos científicos en las bases de datos electrónicas de: PubMed, Lilacs y Scopus, utilizándose los descriptores: "Dental Implant", "Low Level Laser Therapy" y "Osseointegration", indexados en el periodo de 2016 y 2020 que trataban de ensayo clínico, revisiones, metaanálisis, y control aleatorio y fueron excluídos artículos de libros y documentos, documento de sesión, capítulo de libro y editoriales. posteriormente criterios de elegibilidad fueron analizados 15 artículos integralmente publicados en lengua Inglesa. Los estudios mostraron que el uso del láser de baja potencia ayuda a la implantología dental en lo que se refiere a. la osteointegración del implante, disminuyendo el periodo de postoperatorio, dolor y riesgo de inflamación debido a su poder bioestimulante, analgésico y antiinflamatorio. Esta eficacia deriva de un aumento en la proliferación epitelial y de fibroblasto, de un estímulo de la síntesis de colágeno, de la actividad fagocítica y de la inducción de la endorfina. Sin embargo, todavía no existen estudios de casos suficientes, principalmente en humanos, para que se compruebe de hecho, que la utilización del láser auxilie en el proceso de integración entre hueso e implante llamado de "osteointegración", no obstante, es notable que esta técnica se muestra prometedora para el área de la odontología.

Palabras clave: Implante dental; Terapia a laser de bajo nivel; Osteointegración.

\section{Introdução}

O aprimoramento da utilização de tecnologias a laser, como a compreensão da interação luz-tecido, permitiu grandes avanços na medicina e na odontologia, sendo o uso desses lasers, de alta ou baixa potência, comum em diversos processos. Contudo, a terapia de baixa intensidade (LLT), conhecida também como biomodulação (PMB), ainda deve ser mais utilizada na odontologia. (Zayed et al, 2020).

Os implantes dentais são bastantes utilizados em pessoas que desejam substituir dentes perdidos ou com falhas, e para se obter boa estabilidade desse implante é preciso que o processo de osseointegração apresente bons resultados. A terapia a laser de baixa intensidade (LLLT) apresenta uma resposta significativa na melhoria dessa estabilidade primária nas fases iniciais da osseointegração (Lobato et al, 2020).

A fototerapia do laser ocorre devido ao comprimento de onda infravermelho que é absorvido pelo tecido, o que estimula ao redor do local da aplicação várias respostas, como a atividade osteoblástica, bioestimulando a formação do tecido ósseo e redução de processos inflamatórios, essa LLLT também está relacionada com a penetração da luz no comprimento de onda 
(Lobato et al, 2020). Além disso, a laserterapia tem auxiliado a implantodontia oral a reduzir o tempo de cicatrização (Zayed et al, 2020).

Desta forma, o objetivo do presente estudo é realizar uma revisão integrativa da literatura para investigar a eficácia da terapia a laser de baixa intensidade guiada na realização de implantes dentários em pacientes com a falta desse dente ou que precisem substituir dentes com falhas, destacando seus benefícios, limitações, relevância e impacto para a saúde bucal e sociedade.

\section{Metodologia}

\subsection{Estratégia de busca}

O presente estudo trata-se de uma revisão integrativa da literatura, realizada por meio de uma busca de artigos científicos nas bases de dados eletrônicos PubMed, Lilacs e Scopus utilizando os descritores "dental implant”, "low level laser therapy” e "osseointegration" combinados com as associações e desfechos de interesse foram elaborados entre os descritores descrita abaixo na Figura 1.

Figura 1. Fluxograma de estratégia de busca nas bases de dados.

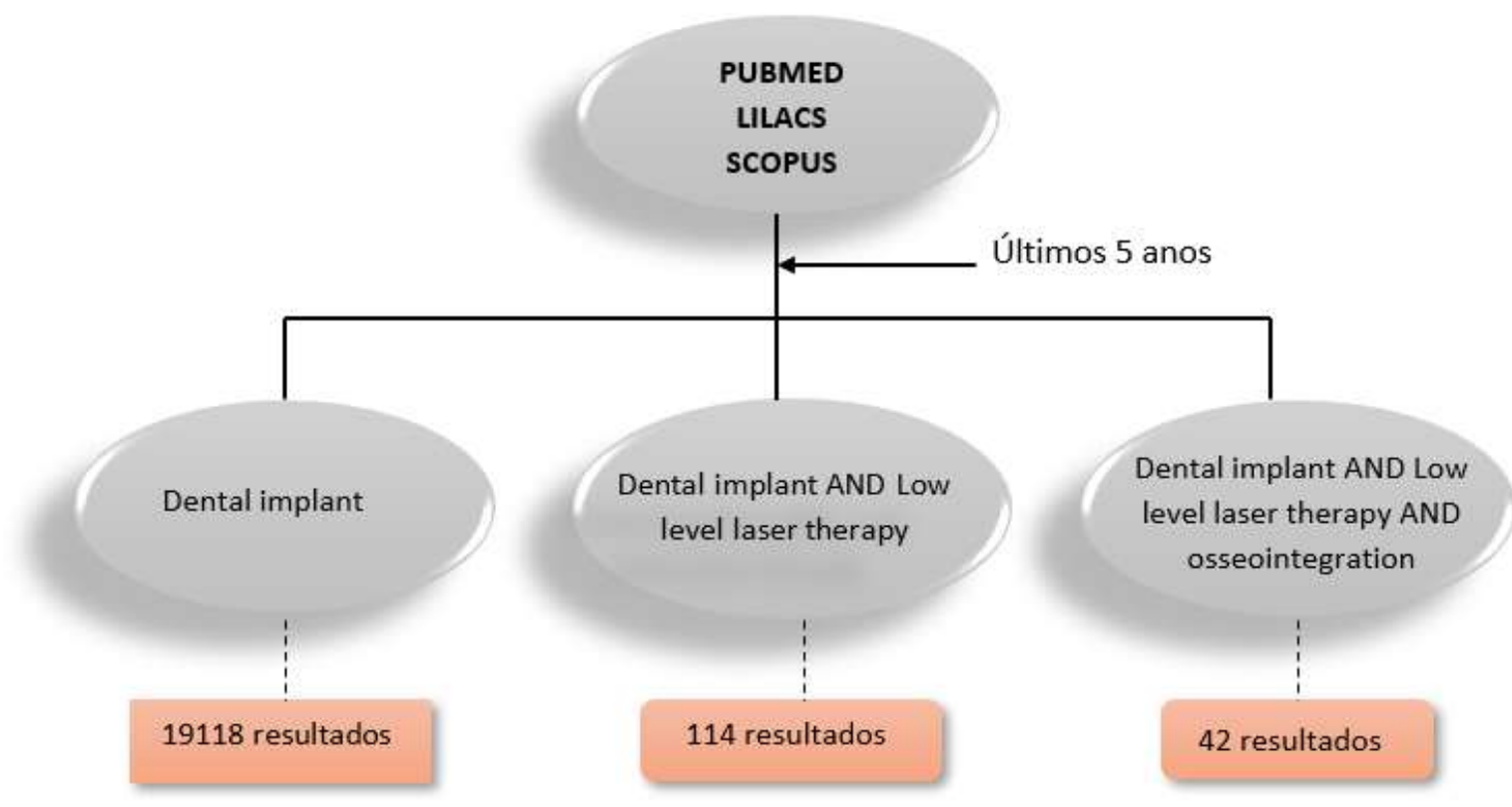

Fonte: Autores (2020).

A partir da busca de artigos científicos, nas plataformas acima mencionadas, no período de 2016 a outubro de 2020 , utilizando o descritor "dental implant” obtivemos um total de 19.118 artigos, após a junção desse descritor com "low level laser therapy" foi filtrado 114 resultados que já apareciam na pesquisa com apenas o primeiro descritor. Após a junção dos descritores anteriores mais “osseointegration” obtivemos uma amostra de 42 artigos, também, já apresentados nas duas outras pesquisas. 


\subsection{Seleção das publicações e extração dos dados}

O processo de análise para avaliação e seleção dos artigos foi realizado por dois pesquisadores (A.S., R.N.), de forma independente, com posterior confronto dos resultados para obtenção dos textos selecionados por consenso. Em casos de divergências ou dúvidas quanto à inclusão dos artigos, houve a participação de um terceiro pesquisador-avaliador (M.M.). Os artigos indexados repetidamente nos dois bancos de dados foram considerados apenas uma vez. Então, a seleção das publicações foi conduzida em duas fases: $1^{\text {a }}$ : leitura dos resumos, e $2^{\mathrm{a}}$ : análise qualitativa dos textos na íntegra.

\subsection{Critérios de elegibilidade do artigo}

Para a seleção das fontes, foram consideradas como critérios de inclusão dos artigos originais disponibilizados na íntegra, publicados em língua inglesa, língua portuguesa e língua espanhola indexados no período de 2016 a outubro de 2020 que tratavam de "clinical trial", "review", "systematic review", "meta-analysis" e "randomized controlled". Não foram incluídos artigos de "books and documents", "confere paper", "book chapter" e editoriais.

Segue a Figura 2, que ilustra a estratégia de busca de acordo com os critérios citados.

Figura 2. Fluxograma de estratégia de busca.

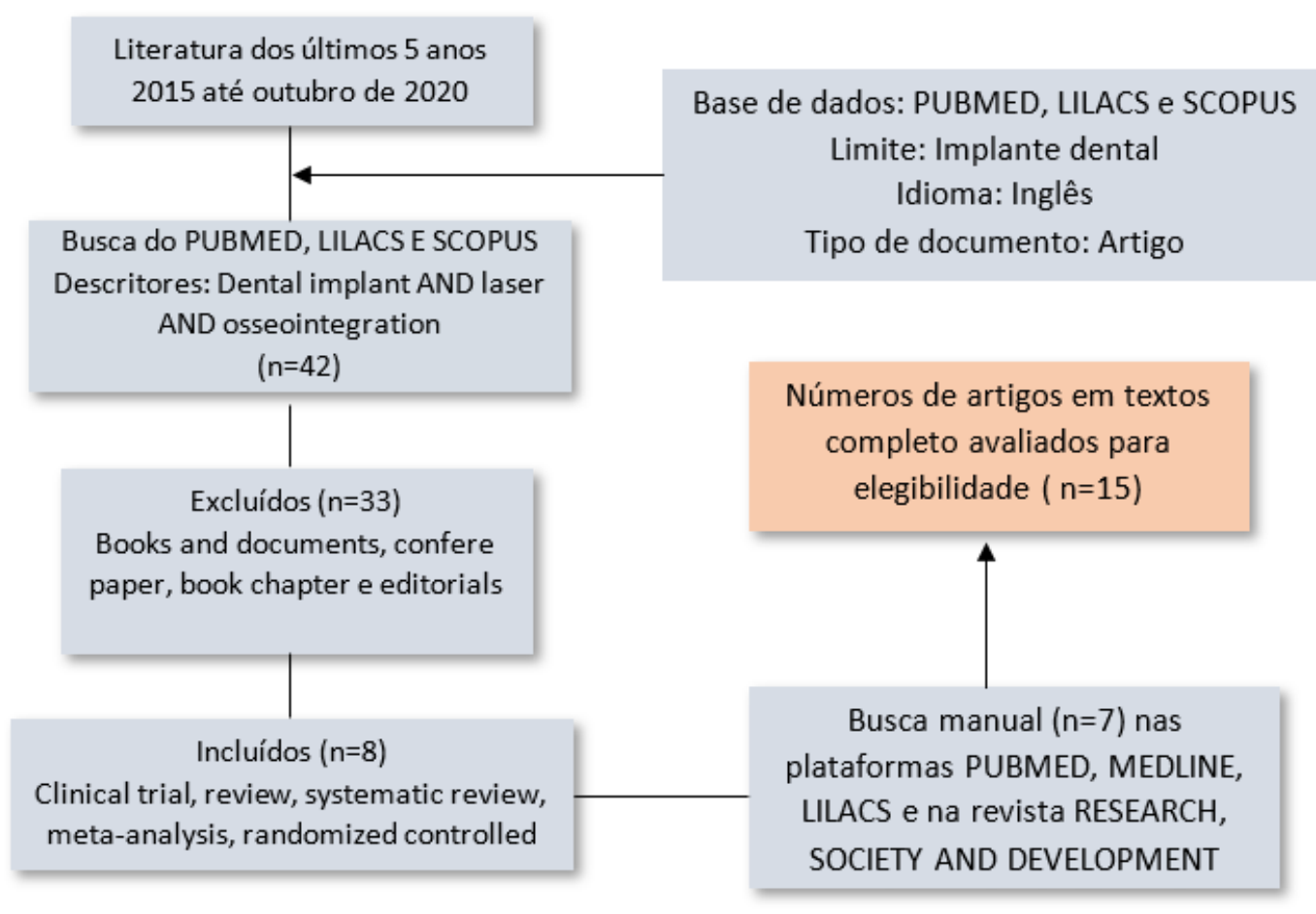

Fonte: Autores (2020).

Esta metodologia seguiu as recomendações PRISMA (principais itens para relatar revisões sistemáticas e metanálise). Os artigos que não se encaixavam nos critérios de inclusão, ou repetidos, foram excluídos. Ao todo foi encontrado um total de 1 artigo repetido, sendo esse o artigo "Influence of low-level laser therapy on implant stability in implants placed in fresh extraction sockets: A radomized clinical trial" Lobato R. P. B. (2019), compartilhado pela plataforma pubmed e scopus. Além disso, houve a busca manual de sete artigos, dois encontrados no Medline, um no Lilacs, um no Pubmed e três na revista Research, Society and Development, os quais foram considerados elegíveis e incluídos no trabalho. Posteriormente, dos artigos, 
restantes, foi realizada a leitura completa, chegando a uma amostra final de 15 (quinze) artigos incluídos nesta revisão. A presente literatura busca explicar os benefícios, limitações, desafios, relevâncias e impactos para saúde bucal e para sociedade do uso do laser na implantodontia.

Não foram utilizadas teses, dissertações e monografias, visto que a realização de uma busca desses estudos é inviável logisticamente e a base de dados utilizada não tem esse tipo de publicação.

\section{Resultados e discussão}

\subsection{Resultados}

Após colocar os descritores nas bases de dados do Pubmed, Lilacs e Scopus, foram encontrados 49 artigos, 8 desses artigos foram considerados elegíveis, 1 artigo duplicado foi removido e 7 artigos foram encontrados através de uma busca manual, chegando ao final com um total de 15 artigos que atenderam aos critérios de elegibilidade. A presente revisão integrativa selecionou esses estudos recentes que demostram como o uso do laser de baixa potência é capaz de influenciar positivamente na interação do osso com o implante dentário, acelerando, dessa forma, o período de osseointegração, como mostra no Quadro 1. 
Quadro 1. Seleção dos artigos de acordo com o autor, tipo de estudo, objetivos, metodologia, resultados e conclusão.

\begin{tabular}{|c|c|c|c|c|}
\hline $\begin{array}{l}\text { Autor e } \\
\text { Estudo }\end{array}$ & Objetivo & Metodologia & Resultados & Conclusão \\
\hline $\begin{array}{l}\text { Lobato R. P. } \\
\text { B. } \\
(2019) \\
\text { Randomized clinical } \\
\text { trial }\end{array}$ & $\begin{array}{l}\text { Para avaliar a influência do } \\
\text { LLLT na estabilidade do } \\
\text { implante em implantes } \\
\text { colocados em encaixes de } \\
\text { extração frescas. }\end{array}$ & $\begin{array}{l}\text { Os pacientes foram } \\
\text { aleatoriamente alocados de acordo } \\
\text { com controle ou grupos LLLT } \\
\text { aplicado em modo de contato com } \\
\text { tecidos moles peri-implantar } \\
\text { antes da perfuração óssea e após a } \\
\text { sutura. O protocolo LLLT foi } \\
\text { aplicado apenas na sessão de } \\
\text { colocação de implante dentário. }\end{array}$ & $\begin{array}{l}50 \text { implantes foram colocados em } 44 \\
\text { pacientes. Nenhuma diferença foi } \\
\text { observada quando comparado os } \\
\text { grupos com diferença de ISQ ou peri- } \\
\text { implantar radiográfico alterações. }\end{array}$ & $\begin{array}{l}\text { LLLT não influenciou a estabilidade do } \\
\text { implante em implantes colocados em } \\
\text { soquetes de extração quando avaliados na } \\
\text { instalação do pilar de } \\
\text { cicatrização. }\end{array}$ \\
\hline $\begin{array}{l}\text { Prados-Fruto } \\
\text { J.C. } \\
2016 \\
\text { Review article }\end{array}$ & $\begin{array}{l}\text { O objetivo deste estudo foi } \\
\text { avaliar o efeito da LLLT na } \\
\text { interação entre o osso e o } \\
\text { implante dentário de titânio e a } \\
\text { qualidade metodológica dos } \\
\text { estudos. }\end{array}$ & $\begin{array}{l}\text { Incluímos artigos que } \\
\text { foram publicados a partir de } \\
\text { janeiro de } 2000 \text { a } 2015 \text { e foram } \\
\text { estudos in vitro e in vivo que } \\
\text { avaliaram o efeito da LLLT na } \\
\text { osseointegração de implantes de } \\
\text { titânio. }\end{array}$ & $\begin{array}{l}\text { Todas as estratégias de busca resultaram } \\
\text { em } 37 \text { artigos. Dois } \\
\text { Investigadores identificaram } \\
\text { Independentemente } 18 \text { artigos elegíveis. } \\
\text { A concordância entre examinadores } \\
\text { sobre a elegibilidade do estudo foi alta. }\end{array}$ & $\begin{array}{l}\text { Não há clínicas suficientes ou estudos que } \\
\text { analisam os efeitos desse tratamento na } \\
\text { osseointegração dos implantes em humanos. } \\
\text { De acordo com os resultados da pesquisa } \\
\text { experimental, LLLT pode ser útil para o } \\
\text { processo de osseointegração. }\end{array}$ \\
\hline $\begin{array}{l}\text { Zayed S.M. } \\
2020 \\
\text { Systematic } \\
\text { Review }\end{array}$ & $\begin{array}{l}\text { O objetivo era avaliar a } \\
\text { eficácia clínica do PBM na } \\
\text { osseointegração de } \\
\text { implantes dentários }\end{array}$ & $\begin{array}{l}\text { Foi realizada uma busca } \\
\text { eletrônica de forma independente } \\
\text { de artigos publicados desde o } \\
\text { início até janeiro de } 2020 \text {, nos } \\
\text { bancos de dados PubMed, } \\
\text { Cochrane Library e Embase. } \\
\text { Clínica randomizada ensaios e } \\
\text { estudos clínicos não } \\
\text { randomizados foram incluídos. } \\
\text { Estudos em animais e estudos in } \\
\text { vitro foram excluídos. Apenas } \\
\text { uma síntese narrativa dos estudos } \\
\text { incluídos é apresentada. }\end{array}$ & $\begin{array}{l}\text { Quatro estudos descobriram que o } \\
\text { PBM tem um potencial efeito positivo } \\
\text { sobre o resultado da estabilidade do } \\
\text { implante dentário, enquanto três } \\
\text { estudos relataram que o PBM não tem } \\
\text { efeito sobre o implante e } \\
\text { estabilidade. }\end{array}$ & $\begin{array}{l}\text { A aplicação pós-operatória de PBM pode } \\
\text { potencialmente ter algum efeito positivo na } \\
\text { osseointegração e estabilidade do implante } \\
\text { dentário. No entanto, estudos são necessários } \\
\text { nos métodos para fornecer uma avaliação } \\
\text { mais robusta desse efeito. }\end{array}$ \\
\hline
\end{tabular}




\begin{tabular}{|c|c|c|c|c|}
\hline $\begin{array}{l}\text { Soares, G. P. et al. } \\
\text { (2013) } \\
\text { Clinical trial }\end{array}$ & $\begin{array}{l}\text { Avaliar a influência da } \\
\text { fototerapia a laser na formação } \\
\text { óssea ao redor de implantes de } \\
\text { titânio inseridos em blocos de } \\
\text { diferentes tipos de enxertos } \\
\text { ósseos. }\end{array}$ & $\begin{array}{l}\text { Vinte e quatro coelhas } \\
\text { adultas foram usadas neste estudo. } \\
\text { Após sedação intramuscular (1) e } \\
\text { anestesia geral (2), foram } \\
\text { realizadas duas fases cirúrgicas. }\end{array}$ & $\begin{array}{l}\text { A comparação entre os enxertos } \\
\text { irradiados demonstrou maior } \\
\text { porcentagem de formação de osso. }\end{array}$ & $\begin{array}{l}\text { A comparação dos enxertos não-irradiados e } \\
\text { irradiados mostra que a irradiação aumentou } \\
\text { significativamente o VO para ambos os tipos } \\
\text { de enxertos. }\end{array}$ \\
\hline $\begin{array}{l}\text { Faleiros, P. L. } \\
(2016) \\
\text { clinical trial }\end{array}$ & $\begin{array}{l}\text { Avaliar a influência da terapia } \\
\text { com laser em baixa } \\
\text { intensidade na osseointegração } \\
\text { de implantes instalados em } \\
\text { tíbias de ratos modificados ou } \\
\text { não } \\
\text { sistemicamente pela nicotina. }\end{array}$ & $\begin{array}{l}\text { Um total de } 120 \text { ratos foram } \\
\text { distribuídos em dois grandes } \\
\text { grupos, ocorridos a duas } \\
\text { aplicações solução de solução } \\
\text { salina ou nicotina. Após } 30 \text { dias, } \\
\text { implantes de titânio foram } \\
\text { instalados na metáfise proximal } \\
\text { de ambas as tíbias de todos os } \\
\text { animais. }\end{array}$ & $\begin{array}{l}\text { Por enquanto a nicotina atrasou a } \\
\text { produção de elementos da matriz óssea, } \\
\text { alterou o padrão de imunomarcação e } \\
\text { aumentou a quantidade de células } \\
\text { imunorreativas, o laser influenciou } \\
\text { positivamente a BA e o torque de } \\
\text { remoção dos implantes, aumentou a } \\
\text { angiogênese e a diferenciação } \\
\text { osteoblástica, promovendo a formação } \\
\text { óssea, biomineralização e maturação } \\
\text { óssea peri-implantar nos animais } \\
\text { modificados sistemicamente } \\
\text { pela nicotina. }\end{array}$ & $\begin{array}{l}\text { A LLLT é capaz de promover o processo de } \\
\text { reparo ósseo periimplantar em condições } \\
\text { normais e compensar os efeitos negativos da } \\
\text { nicotina na osseointegração. }\end{array}$ \\
\hline $\begin{array}{l}\text { Yucesoy, T. et al. } \\
\text { (2019) } \\
\text { clinical trial }\end{array}$ & $\begin{array}{l}\text { Avaliar como as forças } \\
\text { ortopédicas pesadas contínuas } \\
\text { afetam a estabilidade de } \\
\text { implantes e a cicatrização do } \\
\text { tecido ósseo circundante com } \\
\text { tratamento de } \\
\text { fotobiomodulação e terapia de } \\
\text { ozônio. }\end{array}$ & $\begin{array}{l}\text { Os implantes foram aplicados nas } \\
\text { tíbias de coelhos que foram } \\
\text { divididos aleatoriamente em três } \\
\text { grupos: controle, } \\
\text { fotobiomodulação e } \\
\text { ozonioterapia. Várias análises } \\
\text { foram realizadas para medir o } \\
\text { nível de quociente de estabilidade } \\
\text { do implante, volume ósseo e } \\
\text { contato osso-implante }\end{array}$ & $\begin{array}{l}\text { Os grupos de fotobiomodulação e } \\
\text { terapia de ozônio revelaram pontuações } \\
\text { significativamente mais altas do que o } \\
\text { grupo de controle no Tempo de } \\
\text { carregamento de } 8 \text { semanas. }\end{array}$ & $\begin{array}{l}\text { Revela um melhor processo de } \\
\text { osseointegração em implantes de mini- } \\
\text { implantes quando tratados com } \\
\text { fotobiomodulação e ozonioterapia em } \\
\text { comparação com grupos controle. }\end{array}$ \\
\hline $\begin{array}{l}\text { Mastrangelo, F. et al. } \\
\text { (2020) } \\
\text { review }\end{array}$ & $\begin{array}{l}\text { Comparar como duas } \\
\text { superfícies de titânio a laser e } \\
\text { superfícies jateadas e } \\
\text { condicionadas }\end{array}$ & $\begin{array}{l}\text { Os osteoblastos humanos foram } \\
\text { cultivados em duas superfícies } \\
\text { diferentes de titânio obtidas a } \\
\text { laser e comparadas com uma } \\
\text { superfície com ácido como }\end{array}$ & $\begin{array}{l}\text { Os osteoblastos mostraram valores } \\
\text { crescentes de mRNA de ALP e } \\
\text { expressão de proteínas. Após } 20 \text { dias de } \\
\text { cultura em amostras de titânio, a } \\
\text { avaliação mostrou um pequeno número }\end{array}$ & $\begin{array}{l}\text { A microtopografia e nanotopografia induzida } \\
\text { por laser de superfície mostrou alta } \\
\text { biocompatibilidade com culturas primárias } \\
\text { de osteoblastos humanos e ausência de }\end{array}$ \\
\hline
\end{tabular}




\begin{tabular}{|c|c|c|c|c|}
\hline & $\begin{array}{l}\text { com ácido influenciam o } \\
\text { comportamento dos } \\
\text { osteoblastos humanos durante } \\
\text { a osteogênese e as fases } \\
\text { iniciais da deposição óssea. }\end{array}$ & $\begin{array}{l}\text { controle. O comportamento dos } \\
\text { osteoblastos humanos foi avaliado } \\
\text { com ensaio de viabilidade de } \\
\text { proliferação celular microscopia } \\
\text { eletrônica de varredura. }\end{array}$ & $\begin{array}{l}\text { de osteoblastos humanos em locais } \\
\text { isolados de deposição de matriz óssea. } \\
\text { Ao mesmo tempo, nas superfícies L1, } \\
\text { apenas uma mono-camada de } \\
\text { osteoblasto com deposição óssea inicial } \\
\text { foi encontrada. }\end{array}$ & $\begin{array}{l}\text { impurezas. A textura do laser foi capaz de } \\
\text { influenciar o processo osteogênico. }\end{array}$ \\
\hline $\begin{array}{l}\text { Gumus, K. Ç. et al. } \\
(2020) \\
\text { clinical trial }\end{array}$ & $\begin{array}{l}\text { O objetivo do presente estudo } \\
\text { foi testar a descontaminação } \\
\text { em discos de titânio } \\
\text { contaminados e comparar este } \\
\text { com os métodos de } \\
\text { descontaminação atuais no } \\
\text { tratamento de peri-implantite. }\end{array}$ & $\begin{array}{l}\text { Os métodos usados na literatura } \\
\text { são principalmente mecânicos e } \\
\text { intervenções químicas, bem como } \\
\text { tratamentos a laser. }\end{array}$ & $\begin{array}{l}\text { No grupo de tratamento mecânico, a } \\
\text { superfície era achatada irregularmente. } \\
\text { No grupo de tratamento a laser, a } \\
\text { eliminação da placa ocorreu da mesma } \\
\text { forma que no grupo com fluxo de ar de } \\
\text { longo prazo. }\end{array}$ & $\begin{array}{l}\text { Verificou-se que o tratamento a laser é um } \\
\text { dos métodos mais adequados para } \\
\text { desintoxicar e melhorar as superfícies de } \\
\text { implantes infectados para se tornarem mais } \\
\text { adequados para a adesão de coágulos e } \\
\text { estabilização de feridas. }\end{array}$ \\
\hline $\begin{array}{l}\text { Abousheub, M. N. et } \\
\text { al. } \\
(2016) \\
\text { Clinical management }\end{array}$ & $\begin{array}{l}\text { Propor um protocolo para o } \\
\text { tratamento de candidatos que } \\
\text { sofrem de líquen plano ativo. }\end{array}$ & $\begin{array}{l}\text { Os pacientes receberam implantes } \\
\text { dentários usando a técnica de } \\
\text { retalho aberto e osteotomia } \\
\text { sequencial. Após um tempo de } \\
\text { cicatrização de } 6 \text { semanas, os } \\
\text { implantes foram colocados em } \\
\text { contato } \\
\text { oclusal usando próteses } \\
\text { provisórias. }\end{array}$ & $\begin{array}{l}\text { Os exames de sangue revelaram uma } \\
\text { alta contagem de células CD8 e as } \\
\text { biópsias de tecidos moles revelaram } \\
\text { infiltração de células inflamatórias na } \\
\text { interface de tecido mole do epitélio do } \\
\text { tecido examinado. Ao administrar } \\
\text { corticosteroides orais e irradiação a } \\
\text { laser de tecidos } \\
\text { moles ouve uma melhora acentuada da } \\
\text { mucosa oral após o tratamento. }\end{array}$ & $\begin{array}{l}\text { O líquen plano ativo deve ser tratado com } \\
\text { corticosteroides orais e irradiação a laser de } \\
\text { tecidos moles antes da inserção de implantes } \\
\text { dentários. }\end{array}$ \\
\hline $\begin{array}{l}\text { Mayer, L. et al. } \\
(2013) \\
\text { Clinical trial }\end{array}$ & $\begin{array}{l}\text { Descrever a técnica cirúrgica e } \\
\text { a sequência laboratorial } \\
\text { utilizada na obtenção de dados } \\
\text { por meio de dosagem } \\
\text { hormonal, análise da } \\
\text { frequência de ressonância } \\
\text { (AFR), tomografia } \\
\text { computadorizada de feixe } \\
\text { cônico (TCFC), microscopia } \\
\text { eletrônica de varredura } \\
\text { (MEV), espectrometria por }\end{array}$ & $\begin{array}{l}\text { Foram utilizados } 40 \text { coelhos } \\
\text { machos, distribuídos em cinco } \\
\text { grupos, sendo dois grupos } \\
\text { designados controle e três grupos } \\
\text { designados experimentais. Os } \\
\text { cinco grupos foram submetidos a } \\
\text { coletas sanguíneas para dosagens } \\
\text { de tri-iodotironina (T3), tiroxina } \\
\text { (T4), cálcio e albumina, sendo o } \\
\text { grupo CI considerado controle } \\
\text { hematológico absoluto. Nos }\end{array}$ & $\begin{array}{l}\text { Apresenta-se, pois, um roteiro que pode } \\
\text { servir de modelo para colegas } \\
\text { pesquisadores. }\end{array}$ & $\begin{array}{l}\text { Rotinas técnicas padronizadas para a } \\
\text { realização de cada fase de um experimento } \\
\text { qualificam uma linha de pesquisa de um } \\
\text { grupo constituído com o objetivo de realizar } \\
\text { uma sequência de análises que possam vir a } \\
\text { responder a um conjunto de questões } \\
\text { científicas de uma área delimitada do } \\
\text { conhecimento na especialidade, com ênfase } \\
\text { na laserterapia. Nesse campo do } \\
\text { conhecimento em particular, enfrenta- -se } \\
\text { uma série de dificuldades, devido ao fato de }\end{array}$ \\
\hline
\end{tabular}




\begin{tabular}{|c|c|c|c|c|}
\hline & $\begin{array}{l}\text { dispersão de energia (EDS) e } \\
\text { análise histológica e } \\
\text { histomorfométrica da região } \\
\text { perimplantar pós-terapia com } \\
\text { laser não ablativo (LLLT) em } \\
\text { modelo experimental coelho. }\end{array}$ & $\begin{array}{l}\text { demais grupos, foram realizadas a } \\
\text { extração do incisivo inferior } \\
\text { esquerdo e a colocação de um } \\
\text { implante imediato com aferição } \\
\text { da frequência de ressonância } \\
\text { (AFR). }\end{array}$ & & $\begin{array}{l}\text { as metodologias empregadas não se } \\
\text { apoiarem nessa lógica de rotina. }\end{array}$ \\
\hline $\begin{array}{l}\text { Nagai, T. K. et al. } \\
(2021) \\
\text { Review }\end{array}$ & $\begin{array}{l}\text { O objetivo foi realizar uma } \\
\text { revisão de literatura sobre a } \\
\text { importância da mucosa } \\
\text { queratinizada na } \\
\text { implantodontia; analisando a } \\
\text { relação entre a manutenção } \\
\text { gengival e saúde } \\
\text { periimplantar. }\end{array}$ & $\begin{array}{l}\text { Bases de dados foram acessadas } \\
\text { para realização da pesquisa de } \\
\text { artigos publicados na literatura } \\
\text { Odontológica, na língua } \\
\text { portuguesa e inglesa, } \\
\text { principalmente entre os anos de } \\
2010 \text { a 2019. As bases de dados } \\
\text { acessadas foram: LILACS, } \\
\text { PUBMED, Scielo e a } \\
\text { Biblioteca Digital Brasileira de } \\
\text { Teses e Dissertações (BDTD). } \\
\text { As palavras chaves usadas } \\
\text { foram: osseointegration AND } \\
\text { periodontics AND mouth } \\
\text { mucosa AND gingiva. }\end{array}$ & $\begin{array}{l}\text { A presença da faixa de tecido } \\
\text { ceratinizado não é necessária para a } \\
\text { sobrevivência dos implantes a longo } \\
\text { prazo, isso quando a higiene bucal é } \\
\text { realizada de forma adequada, mas } \\
\text { enfatizaram que a ausência deste tecido } \\
\text { torna a higienização menos confortável } \\
\text { para o paciente. }\end{array}$ & $\begin{array}{l}\text { Conclui-se que o tecido ceratinizado é } \\
\text { fundamental para a saúde periimplantar, } \\
\text { atuando tanto na saúde oral do paciente } \\
\text { reabilitado, quanto na sua estética e } \\
\text { funcionalidade. }\end{array}$ \\
\hline $\begin{array}{l}\text { Gonçalves, S. M. et } \\
\text { al. } \\
(2020) \\
\text { Review }\end{array}$ & $\begin{array}{l}\text { O objetivo do presente estudo } \\
\text { foi analisar a influência do uso } \\
\text { dos BFs no processo de } \\
\text { osteointegração de implantes } \\
\text { dentários. }\end{array}$ & $\begin{array}{l}\text { A estratégia de busca utilizada foi } \\
\text { "Biphosphonates" and } \\
\text { "Osseointegration" and "Oral" } \\
\text { and "Maxillofacial" and } \\
\text { "Sugery" and "Dental } \\
\text { implants". Foram selecionados } \\
30 \text { artigos publicados entre } 2008 \\
\text { até } 2020 \text {, com base nos } \\
\text { seguintes critérios de inclusão: } \\
\text { disponibilidade do texto } \\
\text { integral, publicação nas línguas } \\
\text { portuguesa, inglesa e espanhola, } \\
\text { clareza no detalhamento } \\
\text { metodológico utilizado e artigos }\end{array}$ & $\begin{array}{l}\text { Os resultados obtidos indicaram } \\
\text { uma ocorrência insignificante de } \\
\text { osteonecrose, além de não identificar } \\
\text { uma falha na taxa de sucesso da } \\
\text { osseointegração dos implantes } \\
\text { dentários. A maioria dos estudos } \\
\text { utilizados para realização da revisão } \\
\text { revelou que a osteoporose e o uso de } \\
\text { bifosfonatos não têm efeito sobre a } \\
\text { osseointegração e na função dos } \\
\text { implantes bem sucedidos. }\end{array}$ & $\begin{array}{l}\text { Deve ser avaliado o risco do } \\
\text { desenvolvimento de osteonecrose em } \\
\text { pacientes que fazem o uso de BFs } \\
\text { intravenoso }\end{array}$ \\
\hline
\end{tabular}




\begin{tabular}{|c|c|c|c|c|}
\hline & & $\begin{array}{l}\text { que enfocassem estratégias para } \\
\text { o uso do bisfofonatos em } \\
\text { implantodontia. }\end{array}$ & & \\
\hline $\begin{array}{l}\text { Karaca, I. R. et al. } \\
(2018) \\
\text { Clinical trial }\end{array}$ & $\begin{array}{l}\text { O objetivo deste estudo foi } \\
\text { investigar os efeitos dos lasers } \\
\text { de bioestimulação e da terapia } \\
\text { com ozônio na } \\
\text { osseointegração de implantes } \\
\text { de carga imediata. }\end{array}$ & $\begin{array}{l}\text { Um número total de } 100 \\
\text { implantes foi aplicado a } 25 \\
\text { pacientes uniformemente. Coroas } \\
\text { provisórias foram aplicadas a cada } \\
\text { paciente na mesma sessão da } \\
\text { cirurgia. Os implantes foram } \\
\text { divididos em quatro grupos de } \\
\text { tratamento (Grupo } 1 \text { de terapia a } \\
\text { laser de baixa intensidade } \\
\text { (LLLT), Grupo } 2 \text { de terapia com } \\
\text { ozônio, Grupo } 3 \text { de protocolo } \\
\text { diferente de grupo de terapia de } \\
\text { ozônio e Grupo } 4 \text { de controle, } \\
\text { cada um com } 25 \text { implantes. }\end{array}$ & $\begin{array}{l}\text { Neste estudo, a taxa de sobrevivência } \\
\text { geral do implante foi de } 92 \% \text { após um } \\
\text { período de } 6 \text { meses. Os valores do } \\
\text { quociente de estabilidade do implante } \\
\text { foram encontrados significativamente } \\
\text { maiores no Grupo } 1 \text { (grupo LLLT) e } \\
\text { Grupo } 3 \text { (protocolo diferente do grupo } \\
\text { de terapia com ozônio) do que nos } \\
\text { outros grupos. Não houve diferença } \\
\text { significativa no Grupo } 2 \text { (grupo de } \\
\text { terapia com ozônio) e no controle. }\end{array}$ & $\begin{array}{l}\text { Os resultados sugerem que tanto a LLLT } \\
\text { quanto a terapia com ozônio com tempo de } \\
\text { aplicação prolongado são métodos } \\
\text { promissores para melhorar a cicatrização } \\
\text { óssea em torno de implantes carregados } \\
\text { imediatamente e aumentar a estabilidade do } \\
\text { implante; entretanto, há necessidade de mais } \\
\text { estudos sobre o assunto para que esses } \\
\text { métodos se tornem aplicações rotineiras. }\end{array}$ \\
\hline $\begin{array}{l}\text { Silva et al. } \\
(2021) \\
\text { Review }\end{array}$ & $\begin{array}{l}\text { Avaliar se a ozonioterapia é } \\
\text { eficaz no tratamento da peri- } \\
\text { implantite }\end{array}$ & $\begin{array}{l}\text { O estudo utilizou o acrônimo } \\
\text { PICOT (P: pacientes com peri- } \\
\text { implantite; I: ozônio; C: sem } \\
\text { tratamento ou tratamento sem } \\
\text { ozônio; O: periodonto submetido } \\
\text { a tratamento para peri-implantite; } \\
\text { T: Pré-clínicos in vivo em seres } \\
\text { humanos, e clínicos). }\end{array}$ & $\begin{array}{l}\text { Dentro dos estudos incluídos, um } \\
\text { total de } 156 \text { indivíduos, com } \\
\text { diagnóstico de peri-implantite, em ao } \\
\text { menos um implante dentário, foram } \\
\text { avaliados. Para diagnóstico e análise } \\
\text { do tratamento, foram verificados } \\
\text { parâmetros clínicos, como índice de } \\
\text { placa (IP), índice gengival (IG), } \\
\text { sangramento à sondagem (SS), } \\
\text { profundidade de sondagem (PS), } \\
\text { recessão da mucosa (RM) e nível de } \\
\text { inserção clínica (NIC). }\end{array}$ & $\begin{array}{l}\text { Foi possível concluir que a terapia de } \\
\text { ozônio, com suas poderosas propriedades, } \\
\text { tem grande potencial no tratamento da } \\
\text { peri-implantite, apesar da alta } \\
\text { heterogeneidade e a baixa qualidade dos } \\
\text { estudos inseridos não permitirem uma } \\
\text { evidência de alta qualidade. }\end{array}$ \\
\hline $\begin{array}{l}\text { Mohajerani, H. et al. } \\
(2020) \\
\text { Randomized clinical } \\
\text { trial }\end{array}$ & $\begin{array}{l}\text { O objetivo deste estudo foi } \\
\text { avaliar o efeito do uso } \\
\text { combinado de terapia com } \\
\text { laser de baixa potência (LLL) } \\
\text { e diodo emissor de luz (LED) }\end{array}$ & $\begin{array}{l}\text { Este foi um ensaio clínico } \\
\text { randomizado. Os pacientes foram } \\
\text { divididos em dois grupos. No } \\
\text { grupo 1, os pacientes receberam } \\
\text { LLL e LED } 20 \text { min / dia por } 10\end{array}$ & $\begin{array}{l}\text { Cinquenta e oito pacientes foram } \\
\text { estudados em dois grupos }(\mathrm{n}=28) \text {. O } \\
\text { ISQ médio não diferiu imediatamente } \\
\text { após a inserção }(\mathrm{P}>0,05) \text {. O ISQ médio } \\
\text { diferiu significativamente entre os dois }\end{array}$ & $\begin{array}{l}\text { Os resultados deste estudo mostraram que o } \\
\text { uso simultâneo de LLL e LED aumentou a } \\
\text { estabilidade dos implantes após } 9 \text { semanas } \\
\text { de acompanhamento. }\end{array}$ \\
\hline
\end{tabular}


Research, Society and Development, v. 10, n. 3, e31310313322, 2021

(CC BY 4.0) | ISSN 2525-3409 | DOI: http://dx.doi.org/10.33448/rsd-v10i3.13322

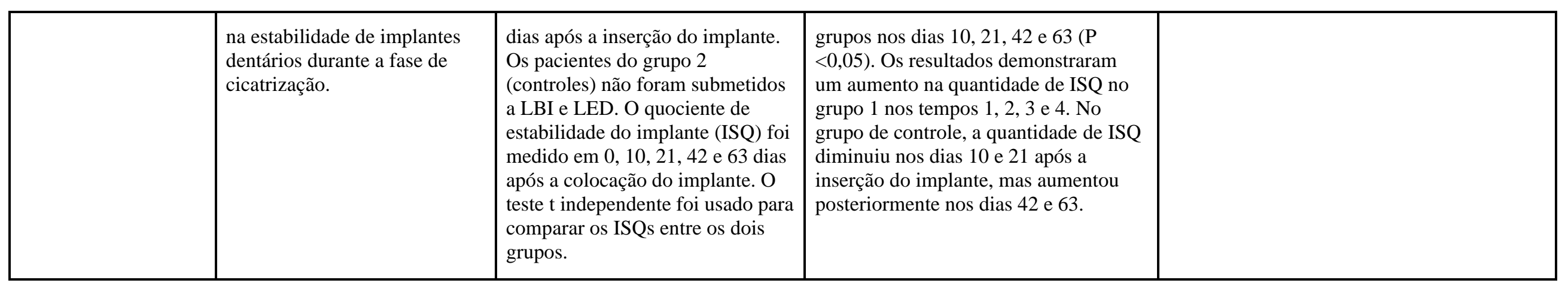

Fonte: Autores (2020). 


\subsection{Discussão}

O implante dental se destaca como um procedimento efetivo e satisfatório na reabilitação oral por ser um método de sucesso a longo prazo que preserva a funcionalidade e a estética bucal do paciente. Contudo, após o implante, existe o processo inflamatório no reparo ósseo perimplantar, o qual pode estar associado à presença de linfócitos, plasmócitos e macrófagos a proliferação de vasos sanguíneos, a fibrose e a necrose tecidual, conforme afirma Mayer et al (2013). Nesse contexto, buscamse cada vez mais alternativas para contornar os desconfortos sentidos pelo paciente, acelerar o processo de osseointegração do implante e evitar os medicamentos convencionais, como os bifosfonatos (BFs), os quais podem trazer efeitos colaterais negativos, como o desequilíbrio na atividade dos osteoclastos e osteoblastos, promovendo uma maior reabsorção do que deposição óssea, de acordo com Gonçalves et al (2020).

A literatura mostra diversas possibilidades para o uso do laser de baixa potência na implantodontia, desde a limpeza da área a sofrer implantação até a efetivação cirúrgica, segundo Prados-Frutos (2016), SOARES et al. (2013) e Abousheub et al. (2016). O uso do LLLT no implante demonstra um grande potencial para a melhoria do processo de osseointegração, de amenizar desconfortos relativos ao pós-operatório e planejamento cirúrgico, conforme Zayed (2020), Faleiros (2016), Yucesoy et al. (2019) e Mastrangelo et al. (2020). Os estudos de Prados-Frutos (2016), Soares et al. (2013) e Abousheub et al. (2016) apresentam inúmeras possibilidades para a aplicação do laser de baixa potência no auxílio do processo de implantodontia, tanto no preparo do tecido periodontal, anterior a fixação da raiz artificial biocompatível, quanto em suas vantagens no pós-operatório. Esses bons resultados são obtidos devido a um aumento na proliferação epitelial e de fibroblastos e estimulação da síntese de colágeno e atividade fagocítica e ao aumento de deposição de hidroxiapatita de cálcio pelos osteoblastos irradiados pela laserterapia de baixa potência, conforme afirma Karaca et al (2018) e Mayer et al (2013).

A aplicação do laser no implante se mostrou eficaz em acelerar o processo osteogênico, descontaminar a área periodontal adjacente ao implante e de compensar efeitos indesejados de substâncias tóxicas, como a nicotina, na área sensível do implante, de acordo com os estudos Mastrangelo et al. (2020), Yucesoy et al. (2019) e Faleiros (2016). Vale ratificar que a saúde dos tecidos adjacentes ao implante determina a osseointegração e promove proteção e reduz inflamações locais, prevenindo recessão gengival, que por sua vez desencadeia resultados estéticos prejudicados, segundo Nagai et al (2021). Além das aplicações práticas já citadas, Yucesoy et al. (2019), Silva et al (2021) e Mohajerani et al (2020) também ressaltam o uso do laser de baixa potência em conjunto com a ozonioterapia, a fotobiomodulação, e o LED (Diodo Emissor de Luz) como uma forma eficiente para inserção de implantes bem fixos e com grande força ortopédica.

Quanto as limitações, Zayed (2020) ressalta alguns resultados contraditórios do uso do laser na osseointegração devido às discrepantes variações nos protocolos de irradiação ou nos modelos experimentais utilizados. Além disso, a distância entre a ponta ativa e o tecido pode influenciar negativamente a eficácia do LLLT, pois uma distância de $10 \mathrm{~mm}$ faz com que uma boa parte do laser seja refletido, o que pode levar também a uma ineficácia do laser. Zayed (2020), também, ressalta que a capacidade do implante de se osteointegrar depende da técnica do operador diretamente de sua estabilidade, para isso a união osso-implante deve ser bem-sucedida, buscando preservar sua integridade estrutural e funcional do local hospedeiro.

Zayed (2020) e Prados-Frutos (2016) apresentam alguns desafios quanto ao uso do LLLT, e, por isso, ressalta a necessidade de mais artigos com estudos clínicos randomizados que com protocolos padrões que mostrem os efeitos potenciais dessas terapias na osseointegração de implantes dentários de titânio.

A aplicação pós-operatória do laser de baixa potência apresenta um potencial de influência clínica positiva na osseointegração e estabilidade de implantes dentários segundo Zayed (2020). Prados-Frutos (2016) relata dois principais tipos de lasers o de alta e o de baixa potência, sendo o de baixa potência os que emitem dentro do espectro vermelho e os mais comuns são o de arseneto de gálio (GaAs) laser de gálio transmissível por fibra óptica e arseneto de alumínio (GaAlAs) e hélio - laser neon $(\mathrm{HeNe})$, este último emitindo dentro do espectro visível, especificamente, o vermelho. 
Gumus et al. (2020), verificou que o laser é um dos métodos mais adequados para descontaminar e melhorar as superfícies de implantes infectados e para se tornarem mais adequados para a adesão de coágulos e estabilização de feridas. Já Mastrangelo et al. (2020), enfatiza que o laser de titânio aumenta a proliferação e diferenciação celular, o que contribui para uma melhor síntese de matriz e deposição óssea.

Visto que cerca de 16 milhões de brasileiros vivem sem os dentes e que 41,5\% da população com mais de 60 anos é totalmente desdentada, segundo pesquisa do Edelman Insights junto ao Instituto Brasileiro de Geografia e Estatística (IBGE), é possível avaliar o impacto do laser de baixa potência no tratamento de implante dental no objetivo de promover a saúde bucal nesse grupo expressivo. É certo que esse paciente desdentado sofrerá complicações como fonética e função mastigatória prejudicadas, além de perturbações psicossociais, e, nesse contexto, o implante do dente perdido será uma intervenção com baixíssimas contra-indicações. Assim, o uso do LLLT beneficiará esse paciente ao lhe oferecer tanto um pré-operatório esterilizado, quanto um pós-operatório seguro e com menores riscos de complicações como infecção e inchaço, garantindo maior estabilidade ortopédica do implante, conforme defende Mastrangelo et al. (2020).

Nosso estudo buscou através de uma revisão integrativa analisar estudos relevantes sobre a aplicação do laser de baixa potência com objetivo de aprimorar a técnica de implante dental, fazendo uma síntese dos conhecimentos relacionados a esse assunto, incluindo os benefícios e limitações, além do impacto na saúde bucal. Esta revisão integrativa se torna relevante, pois encontramos poucos estudos importantes de serem compartilhados com a sociedade e mundo acadêmico. Foram citados os resultados de Zayed (2020) que reitera as múltiplas possibilidades do uso do LLLT e seu efeito positivo na osseointegração de implantes.

O procedimento reparo ósseo peri-implantar foi citado por Faleiros (2016). Já sobre a associação do laser de baixa potência com outras técnicas de fotobiomodulação e ozonioterapia, que aumenta a eficácia da osseointegração, foi citada por Yucesoy et al. (2019).

Importante pontuar que ao colocarmos os descritores Dental implant, Low level laser therapy e osseointegration, escolhidos a partir de uma análise no DECS, foram encontrados trabalhos que não eram compatíveis com o objetivo da revisão integrativa, o que tornou necessária uma filtragem manual para a eliminação de artigos que tangenciam o tema escolhido, artigos que não possuíam informações básicas que agregassem à revisão integrativa e artigos que não estavam integralmente disponíveis para o acesso público.

Por sua vez, ao usarmos os bancos de dados do LILACS, usando os três descritores anteriormente mencionados, foram encontrados 4 artigos, entretanto, 2 desses artigos não estavam completos para leitura e análise. Nessa mesma plataforma de busca, também foi selecionado mais um artigo através de busca manual.

No PUBMED, usando os três descritores, foram encontrados apenas dois artigos e todos eram pertinentes ao tema exposto e ofereciam todas as informações básicas. Além desses, mais um artigo foi selecionado por meio de pesquisa manual.

Já no SCOPUS, usando os três descritores, foram encontrados sete artigos. Entre tais artigos, um foi excluído porque, também, foi publicado no PUBMED e outros dois, porque não estavam disponíveis para leitura.

Outra busca manual foi realizada, na qual foram encontrados mais cinco artigos, dois do MEDLINE e três da revista Research, Society and Development.

Foi importante o uso do conectivo AND para afunilar e obtermos tais resultados, vale salientar que apenas os artigos completos foram incluídos, pois muitos, mesmo possuindo resumo, não ofereciam dados inteiros capazes de enriquecer a pesquisa. Tornou-se relevante que os artigos descrevam com objetividade e clareza como foi realizada a busca e uso das plataformas. Tais sites de buscas, PUBMED, LILACS, MEDLINE e SCOPUS são referências mundiais, o que ajudou na pesquisa realizada. 
Vale ressaltar que ao usarmos os bancos de dados, encontramos dificuldades de como utilizar essas plataformas e de ter acesso aos artigos encontrado, mas que foram solucionadas quando utilizamos o servidor proxy. Observamos que foi fundamental o uso das more, and, filtros de tipos de artigos e delimitação do período dos últimos cinco anos, sendo de 2016 a 2020, para afunilar e obtermos tais resultados.

\section{Considerações Finais}

Por fim, podemos afirmar que a literatura converge quanto à eficácia, praticidade, segurança e aplicabilidade clínica desta técnica. Porém, observamos que é preciso definir e aprimorar um protocolo único que ofereça um resultado mais conclusivo para aplicação do laser na implantodontia, pois, existe uma grande diversidade de metodologias e parâmetros de lasers utilizados. Além disso, nota-se, a eficácia do laser no processo de osseointegração em condições normais na implantodontia, principalmente a aplicabilidade dessa técnica no pós-operatório. Sugere-se, a necessidade de mais trabalhos futuros que foquem em realizar mais testes para acompanhar a eficácia do laser no processo de implante dentário, através de estudos clínicos controlados, principalmente os realizados em humanos, e que busquem definir um protocolo clínico que seja eficiente para o uso do laser na reparação óssea.

\section{Referências}

Aboushelib, M. N. \& Elsafi, M. H. (2015), Clinical Management Protocol for Dental Implants Inserted in Patients with Active Lichen Planus, 26(1), 10.1111 jopr.12379

Faleiros, P. L., (2016) Influência da nicotina na osseointegração de implantes instalados em tíbias de ratos: avaliação biomecânica, histológica, histométrica e imunoistoquímica, http://hdl.handle.net/11449/142006

Gonçalves, S. M., Pinheiro, J. C., Leite, R. B., Vaz, M. M., Silva, G. G., Paiva, D. F. F., Lira, K. B. F., Silva, R. U. O., Silva, T. A. F., Filho, L. G. X., Neto, A. F. R., Silva, R. R. G., Lima, P. M. S., Silva, A. F., Júnior \& E. M. N. (2020), Influencia da utilização dos bifosfonatos na osseointegracão dos implantes dentários, http://dx.doi.org/10.33448/rsd-v9i11.9736

Gümüs , K. Ç., Ustaog 1u, G., Kara, L., Ercan, E., Albayrak, O. \& Tunali, M. (2020), Nano-Hydroxyapatite Airborne-Particle Abrasion System as an Alternative Surface Treatment Method on Intraorally Contaminated Titanium Discs, 40(4), 10.11607/prd.4852

Karaca, I. R., Ergun, G. \& Ozturk, D. N. (2018), Is Low level Laser Therapy and Gaseous Ozone Application Effective on Osseointegration of Immediately Loaded Implants? 10.4103/njcp.njcp_82_17

Lobato,R. P. B., Kinalski, M. A., Martins, T. M., Agostini, B. A., Bergoli, C. D. \& Santos, M. B. F. (2020), Influence of low-level laser therapy on implant stability in implants placed in fresh extraction sockets: A randomized clinical trial, 22(3), 10.1111/cid.12904

Mastrangelo, F., Quaresima, F., Canullo, L., Scarano, A., Muzio, L. \& Piattelli, A. (2020) Effects of Novel Laser Dental Implant Microtopography on Human Osteoblast Proliferation and Bone Deposition, 35(2), 10.11607/jomi.7606

Mayer, L., Oliveira, M. G., Massotti, F. P., Gomes, F. V., Guyoti, V., González, F. H. D. \& Weber, J. B. B. (2013), Methodology protocol for assess systemic and local effects of LLLT on osseointegration of dental implants in the rabbits jaws, http://revodonto.bvsalud.org/scielo.php?script=sci_arttext\&pid=S141340122013000200020

Mohajerani H., Salehi, A, M., Shafiei, F. T. S. \& Tabrizi, R. (2019), Can Low-Level Laser and Light-Emitting Diode Enhance the Stability of Dental Implants?, https://doi.org/10.1007/s12663-019-01210-3

Nagai, T. K., Santos, A. M. S., Machado, N. E. S., Mendes, B. C., Neto, T. J. L., Vasques, A. M. V., Júnior, E. D. \& Faveran, L. P. (2021), Importância do tecido ceratinizado para o sucesso na implantodontia, http://dx.doi.org/10.33448/rsd-v10i2.12202

Prados-Frutos, J. C., Rodríguez-Molinero, J., Prados-Privado, M., Torres, J. H. \& Rojo, R. (2016), Lack of clinical evidence on low-level laser therapy (LLLT) on dental titanium implant: a systematic review, 10.1007/s10103-015-1860-0

Silva, C. A., Pereira, T. S., Quirino, E. C. S., Ibiapina, I. M. P. \& Fernandes, A. U. R. (2021), Análise da eficácia da ozonioterapia no tratamento da periimplantite: uma revisão de escopo, http://dx.doi.org/10.33448/rsd-v10i1.11465

Soares, L. G. P., Júnior, E. B. M., Magalhães, C. A. B., Ferreira, C. F., Marques, A. M. C. \& Pinheiro, A. L. B. (2013), New Bone Formation around Implants Inserted on Autologous and Xenografts Irradiated or not with IR Laser Light: A Histomorphometric Study in Rabbits, 24 (3), https://doi.org/10.1590/01036440201302186

Yücesoy, T., Seker, E. D., Cenkcı, E., Yay, A. \& Alkan, A. (2019), Histologic and Biomechanical Evaluation of Osseointegrated Miniscrew Implants Treated with Ozone Therapy and Photobiomodulation at Different Loading Times, 34(6), 10.11607/jomi.7601 
Research, Society and Development, v. 10, n. 3, e31310313322, 2021

(CC BY 4.0) | ISSN 2525-3409 | DOI: http://dx.doi.org/10.33448/rsd-v10i3.13322

Zayed, S. M. \& Hakim, A. A. A. (2020), Clinical Efficacy of Photobiomodulation on Dental Implant Osseointegration: A Systematic Review, 10.4103/sjmms.sjmms_410_19 